\title{
CLASSIFICATION OF MATERIAL SURFACES USING THE POLARIZATION OF SPECULAR HIGHLIGHTS
}

\author{
Lawrence B. Wolff ${ }^{1}$ \\ Computer Science Department \\ Columbia University \\ New York, N.Y. 10027 \\ Columbia University Technical Report CS-333-88 \\ May 1988
}

\section{ABSTRACT}

Recently there has been interest, in computer vision research, in the segmentation of images based upon the actual material makeup of the objects or object parts that constitute image regions. The idea is to identify image characteristics which can be used to predict the material properties of objects that are being imaged. A majority of object surfaces can be simply classified according to their basic electrical properties; metal objects (e.g. Aluminum, Copper) conduct electricity rather well while dielectric objects (e.g. Rubber, Plastic, Ceramic) conduct electricity poorly. Distinguishing image regions according to whether they correspond to metal or dielectric material can provide important information for scene understanding especially in industrial machine vision. One such major application is circuit board inspection where the presence of dielectric or metal material in the wrong place can cause trouble.

A previous approach to the problem of identifying metal or dielectric material in images is based upon careful spectral (i.e. color) analysis of reflected light from material objects. This paper presents a technique for identifying the material properties of objects in an image using a polarizing lens (i.e. Polaroid filter). Two images of the same scene are taken with a polarizing lens placed in front of a camera in two different respective orientations. Effectively these two images represent two linearly independent polarization components of the reflected light. It is shown that when the linearly independent components of polarization are taken parallel and perpendicular with respect to the plane in which specular rays travel that dielectric objects can be distinguished from metallic objects when specular highlights are present. In particular the two polarization components are very similar at specular highlights on metals while the two polarization components for specular highlights on dielectrics are very different, the perpendicular component having much larger magnitude than the parallel component. This is shown to hold regardless of whether the surface is polished or rough. Results for coated surfaces will be presented at a future date.

\footnotetext{
${ }^{1}$ This work was supported in part by ARPA grant \#N00039-84-C-0165 and NSF grant IRI-88-00370. This work was supported in part by an IBM Graduate Fellowship A ward.
} 


\section{INTRODUCTION}

Most object surfaces fall within one of two broad material classes; that of metals or dielectrics. Metals are characterized as having a relatively low electrical resistivity and thus conduct electricity. By definition. dielectric materials are insulators and do not conduct electricity. The difference in the electrical properties of metals and dielectrics in turn produces a different interaction with light, and thus the reflective characteristics of metals and dielectrics can be vastly different. Human vision uses cues such as "shininess" or "specularity" to discern metals from dielectrics. Metals are mostly "shiny" and "specular" while dielectrics tend to have a "dull" luster. As sophisticated as the human visual system appears to be, humans can be easily fooled into thinking that a certain dielectric is a metal, and vice versa. This is true of many of the paints that are out on the market today.

The identification of materials as being a dielectric or a metal is important to a variety of image understanding tasks that build descriptions of objects in a scene. Deciding the identification or even the functionality of a complex object structure built from many parts can be greatly aided by knowing the material makeup of each part. The ability to segment regions and objects within an image can be greatly enhanced by being able to resolve dielectric surfaces from metal surfaces. For instance, adjacent dielectric and metal object regions can look very similar, and therefore without knowledge of material makeup be interpreted as the same object. This can be very important in an application such as circuit board inspection.

It is postulated in [Healey and Binford 1988] and [Healey and Blanz 1988] that it may be possible to distinguish metals and dielectrics from the spectral content of reflected light assuming that the spectral output of the light source is known. While theoretically provoking, these papers do not propose an actual definitive algorithm to distinguish metals and dielectrics. It is not clear how much spectral resolution is required to classify a material surface. If accurate measurement within $10 \mathrm{~nm}$ spectral resolution is required, an expensive monochromator will be needed to implement such an algorithm.

The research presented here in this paper demonstrates a well defined technique for distinguishing dielectrics and metals based upon an analysis of the polarization properties of reflected light. Effectively, the degree to which a material surface polarizes initially unpolarized light upon reflection gives information as to whether the surface is a metal or a dielectric. All that is required, besides an imaging camera, is a relatively inexpensive polarizing filter. What's more is that this technique is very robust in the sense that the results are invariant with respect to a variety of different imaging environments. The technique presented in this paper will use only a point light source. The use of extended light sources have additional advantages, and this will be presented at a future date. The only constraint using a point light source is that the phase angle ${ }^{2}$ be within the range about $90^{\circ}$ to $130^{\circ}$ (a larger range of phase angles is feasible with less accuracy). Otherwise no knowledge is required about imaging geometry whatsoever. That is, other than satisfying the above phase angle constraint, the results of the technique to be presented are independent of any knowledge of the viewing or incident illumination vectors as well as the surface orientation of the object in question. The results hold the same regardless of the surface in question being polished or rough. The results of this technique are also independent of knowledge of the color and intensity of the incident source. Furthermore. it is not required to calibrate the imaging camera with respect to measuring absolute reflected radiance from the object. It will be shown below that it is only required to know the relative magnitudes of the perpendicular and parallel components of polarization with respect to the plane in which specularly reflected light rays travel. A simple technique to evaluate the radiance that image gray level values represent relative to

\footnotetext{
${ }^{2}$ The phase angle is the angle between the incident orientation of a point light source and the viewing vector.
} 
one another is also presented.

\section{POLARIZING PROPERTIES OF MATERIAL SURFACES}

All light possesses a state of polarization which can be resolved into two independent component directions within the plane perpendicular to the direction of propagation. The magnitude of these two polarization components can be resolved by transmitting the light through a linear polarizer oriented in these respective directions. Light which is unpolarized will have equal transmittance through a linear polarizer regardless of its orientation. Such light is emitted by most typical lamps. Light which is partially polarized has a different transmittance for different orientations of a linear polarizer. Such light results from lamp light which is reflected off of material surfaces.

When initially unpolarized light is specularly reflected off of a surface, the reflected light becomes slightly polarized towards the normal to the plane in which the specularly reflected light rays travel. ${ }^{3}$ This can be observed as a slight increase in the transmitted radiance when light reflected from a surface is passed through a linear polarizer which is rotated from the parallel to the perpendicular orientation relative to the specular plane of incidence. We now examine the details of this phenomenon.

Material surfaces are assumed to have a microscopic level of detail which consists of a statistically large distribution of specularly reflecting planar microfacets. By definition, each planar microfacet is perfectly smooth (planar). Reflected light arises from three phenomena:

- Light rays which specularly reflect off a planar microfacet a single time.

- Light rays which go through multiple (at least two) specular reflections off planar microfacets.

- Light rays which penetrate into the top layer of the material surface and then are reflected back out.

These phenomena are illustrated graphically in figure 1.

The component of reflection which arises from the first phenomenon will be called the specular component of reflection. Note that this definition of specular reflection does not make assumptions about the surface normal because it deals with microfacets who's orientation is independent of the surface normal. ${ }^{4}$

The components of reflection which arises from the second and third phenomena will be combined and called the diffuse component of reflection. It is assumed that the diffuse component of reflection is almost completely unpolarized due to the randomization of multiple reflections. ${ }^{5}$ The polarization of the specular component of reflection is dictated by the Fresnel reflection coefficient for the material surface.

In [Wolff 1987], a general theory explaining how light from a point source is polarized upon reflection from material surfaces is derived from the work of [Torrance and Sparrow 1967] and standard physics references

\footnotetext{
${ }^{3}$ This plane is referred to as the specular plane of incidence.

* To be more precise, each microfacet has a random orientation distributed as some function $P(\alpha)$ where $\alpha$ is the angle between the normal to a planar microfacet and the normal to the surface. In general, $P(\alpha)$ will depend on the local surface normal, so the microfacet orientation is not independent of the normal. However, depending on the distribution, one may be able to obtain significant specular highlights (i.e., find a larger number of specularly reflecting microfacets) even when the surface normal is far from bisecting the incident light angle.

${ }^{3}$ The validity of this assumption depends on the material composition (certain crystals are known to polarize the light of their body component) and macroscopic features (e.g., long macroscopic groves may increase the likelihood of low onder multiple reflections and may an impart a polarization to these reflections.)
} 
such as [Born and Wolf 1981]. The consequences of this theory provide the theoretical starting point for the classification techniques presented in this paper.

The model presented in [Wolff 1987] has numerous aspects, many of which deal with how radiance is dependent on imaging geometry. The work presented in this paper is independent of any of the functional relationships between the diffuse and specular components of reflection, and imaging geometry. In fact, this work need not assume the Torrance-Sparrow reflectance model at all. All that is assumed is a reflectance model that consists of the three reflection phenomena listed above and two additional assumptions about the polarization of the diffuse and specular components of reflection, respectively. Furthermore, as will be shown below, the absolute magnitude of the total reflection from the surface never needs to be known. Only the relative magnitudes of the two polarization components of reflection needs to be derived. This makes the required radiometric calibration of the imaging camera much simpler.

The following pair of equations relate the parallel and perpendicular components of polarization respectively to the magnitudes of the diffuse and specular reflection components:

$$
\begin{aligned}
& \frac{1}{2} I_{d}+\left[\frac{F_{\perp}}{F_{\| 1}+F_{\perp}}\right] I_{s}=k_{\perp} \\
& \frac{1}{2} I_{d}+\left[\frac{F_{\|}}{F_{\|}+F_{\perp}}\right] I_{s}=k_{\|}
\end{aligned}
$$

The symbols $I_{d}$ and $I_{s}$ represent the magnitudes of the diffuse and specular components of reflection respectively. These equations assume that the diffuse component of reflection is always unpolarized and that the state of polarization of the specular component is determined by the relative sizes of the Fresnel reflection coefficients $F_{\|}$and $F_{\perp}$. The symbols $k_{\perp}$ and $k_{\|}$represent the transmitted radiance of reflected light through a polarizer oriented perpendicular and parallel to the specular plane of incidence respectively. ${ }^{6}$

The symbols $F_{\|}$and $F_{\perp}$ are the Fresnel reflection coefficients for parallel and perpendicular polarizations, respectively, relative to the specular plane of incidence. It is these reflection coefficients, varying between 0 and 1 inclusive, that determine the magnitudes of the reflected perpendicular and parallel components of polarization. The Fresnel reflection coefficients only depend upon the specular angle of incidence ${ }^{7}, \Psi^{\prime}$. (see figure 2) and the (complex) index of refraction $\eta=n-\kappa i$ which is dependent on material properties. The coefficient of extinction, $\kappa$, is zero for dielectrics in which case $\eta$ is real. In terms of $\Psi^{\prime}$ and $\eta$, the Fresnel reflection coefficients are given by the following equations:

$$
\begin{gathered}
F_{\perp}\left(\Psi^{\prime}, \eta\right)=\frac{a^{2}+b^{2}-2 a \cos \Psi^{\prime}+\cos ^{2} \Psi^{\prime}}{a^{2}+b^{2}+2 a \cos \Psi^{\prime}+\cos ^{2} \Psi^{\prime}} \\
F_{\|}\left(\Psi^{\prime}, \eta\right)=\frac{a^{2}+b^{2}-2 a \sin \Psi^{\prime} \tan \Psi^{\prime}+\sin ^{2} \Psi^{\prime} \tan ^{2} \Psi^{\prime}}{a^{2}+b^{2}+2 a \sin \Psi^{\prime} \tan \Psi^{\prime}+\sin ^{2} \Psi^{\prime} \tan ^{2} \Psi^{\prime}} F_{\perp}\left(\Psi^{\prime}, \eta\right)
\end{gathered}
$$

where

$$
\begin{gathered}
2 a^{2}=\sqrt{\left(n^{2}-\kappa^{2}-\sin ^{2} \Psi^{\prime}\right)^{2}}+n^{2}-\kappa^{2}-\sin ^{2} \Psi^{\prime}, \\
2 b^{2}=\sqrt{\left(n^{2}-\kappa^{2}-\sin ^{2} \Psi^{\prime}\right)^{2}}-\left(n^{2}-\kappa^{2}-\sin ^{2} \Psi^{\prime}\right)
\end{gathered}
$$

\footnotetext{
${ }^{6}$ Again, we reminder the reader that the specular plane of incidence is determined by the light source vector and the viewing vector. It is independent of the surface orientation. However, the term $I s$ may vary greatly as the surface orientation varies, e.g., for almost perfectly smooth surfaces $I_{s}$ approaches a delta function.

${ }^{7}$ The specular angle of incidence is equal to half the phase angle and is the angle of incidence for specularly reflecting light rays off microfacets.
} 
and where $\Psi^{\prime}$ is the specular angle of incidence (half the phase angle) and $\eta=n-\kappa i$ is the (complex) index of refraction.

The graphs for the Fresnel reflection coefficients for different polarizations are depicted in figure 3 and figure 4 for a dielectric and a metal respectively (note the change in vertical scales). In each graph the index of refraction, $\eta$, is listed and is constant for the graphing of the Fresnel reflection coefficients as a function of the specular angle of incidence from $0^{\circ}$ to $90^{\circ}$. As can be seen $F_{\perp}$ is always greater than or equal to $F_{\|}$. Observing equation 1 this implies that the perpendicular component of polarization is always greater than or equal to the parallel component. The larger $F_{\perp}$ is in proportion to $F_{\|}$, the more the polarization state of the reflected light from the material object is biased toward the perpendicular component. To illustrate this more clearly, equation 1 can be equivalently expressed using the Fresnel ratio $q=F_{\perp} / F_{\|}$as:

$$
\begin{aligned}
& \frac{1}{2} I_{d}+\frac{q}{q+1} I_{s}=k_{\perp} \\
& \frac{1}{2} I_{d}+\frac{1}{q+1} I_{s}=k_{\|}
\end{aligned}
$$

The larger the Fresnel ratio $q=F_{\perp} / F_{\|}$, the larger $k_{\perp}$ is relative to $k_{\|}$(assuming $I_{s}>0$ ). Equations 1 and its equivalent representation as equations 2 can be thought to represent a vector equation in two-dimensions as follows:

$$
\left[\frac{1}{2} I_{d}, \frac{1}{2} I_{d}\right]+\left[\frac{q}{1+q} I_{s}, \frac{1}{1+q} I_{s}\right]=\left[k_{\perp}, k_{\| 1}\right]
$$

where the perpendicular and parallel components of polarization $k_{\perp}$ and $k_{\|}$form a vector which is a linear combination of the polarization vectors for the diffuse and specular components. Regardless of how large the Fresnel ratio, $q$, if the magnitude of the specular component $I$, is small compared to the diffuse component $I_{d}$, the polarization components for the total reflected light will be nearly equal. This is the case far from specular highlights. However at specular highlights $I_{s}$ is usually many times larger than $I_{d}$.

The ratio of the magnitude of the perpendicular polarization component to the magnitude of the parallel polarization component, for initially unpolarized light reflected from metals, ranges between 1.0 and 2.0. When initially unpolarized light is specularly reflected off of a dielectric, the polarization of the reflected light is much more biased towards the normal to the specular plane of incidence, than is for reflected light from metals. This holds especially for phase angles ranging from $90^{\circ}$ to $130^{\circ}$. For this range of phase angles the ratio of the magnitude of the perpendicular polarization component to the magnitude of the parallel polarization component, for initially unpolarized light reflected from dielectrics. is greater than 3.0. The most extreme case of polarization of light by a dielectric occurs when the specular angle of incidence is equal to exactly the Brewster angle. ${ }^{8}$ At this specular angle of incidence initially unpolarized becomes linearly polarized upon specular reflection, oriented along the normal to the specular plane of incidence. In this case the ratio of the magnitude of the perpendicular component of polarization to the parallel component of polarization is infinite.

As can be seen in figures 3 and 4 , within the range of specular angles of incidence from $45^{\circ}$ to $65^{\circ}$, (corresponding to phase angles between $90^{\circ}$ and $130^{\circ}$ ), the Fresnel ratio for the dielectric exceeds that for the metal by a large amount. The lowest Fresnel ratio for the dielectric in this range is 6.5 attained at $45^{\circ}$. The Fresnel ratio is infinite for the dielectric at its corresponding Brewster angle of 60.5 . The Fresnel ratio for the metal varies only between 1.06 and 1.17 in this range of specular angles of incidence.

\footnotetext{
${ }^{8}$ The Brewster angle is the specular angle of incidence at which no parallel component of polarization is reflected.
} 


\section{SEGMENTATION OF METALS AND DIELECTRICS}

Equations 2 describe the perpendicular and parallel components of polarization of reflected light from a material surface, assuming that the incident light is from a completely unpolarized point light source. Rearranging terms in equation 2 and then dividing the first equation by the second equation yields the equality

$$
\frac{F_{\perp}}{F_{\|}}=\frac{k_{\perp}-\left(\frac{1}{2} I_{d}\right)}{k_{\|}-\left(\frac{1}{2} I_{d}\right)}
$$

Note that $\frac{1}{2} I_{d}$ is half the magnitude of the diffuse component of reflection.

Suppose that the observed image irradiance is at a pixel that corresponds to a specular highlight on an object surface. In [Buchanan 1987] it was determined that for materials which exhibit significant highlights, that the diffuse component makes up barely 1 percent of the image irradiance at pixels corresponding to points on the specular highlight. It is clear from experience with a camera in the lab that if the gain control is adjusted to make diffuse reflecting points on objects bright enough to be seen, that the image irradiance of specular highlights in the scene are far beyond the dynamic range of the camera, often to the point where vertical blooming sometimes takes place. This is just to illustrate the vast difference in the image irradiance of diffuse reflecting points and specular highlights.

Since $F_{\perp} \geq F_{\|}, k_{\perp}>(1 / 2) I_{\text {s }}$ and therefore for points on specular highlights $I_{d} \ll k_{\perp}$. If in addition $I_{d} \ll k_{\|}$then:

$$
\frac{F_{\perp}}{F_{\|}} \approx \frac{k_{\perp}}{k_{\|}} .
$$

That is, assuming $I_{d} \ll k_{\|}$, the ratio of the observed image irradiance values at a pixel corresponding to a point on a specular highlight for perpendicular and parallel orientations of a polarizing lens in front of the camera, is a good approximation to the ratio of the Fresnel reflection coefficients for perpendicular and parallel polarization. Furthermore, this ratio is invariant with respect to the local orientation of the surface since the only imaging geometric parameters that the Fresnel reflection coefficients are dependent on is the specular angle of incidence. ${ }^{9}$

The condition $I_{\mathrm{d}} \ll k_{\|}$is true for specular highlights on metals and for specular highlights on dielectrics occurring for a specular angle of incidence far from the Brewster angle. As can be seen from equation 3 and simple arithmetic, the ratio $k_{\perp} / k_{\|}$always theoretically underestimates the true Fresnel ratio. In the case of a dielectric where the specular angle of incidence is close to the Brewster angle

$$
\frac{F_{\perp}}{F_{\|}} \gg \frac{k_{\perp}}{k_{\|}}
$$

For all dielectrics with $n=1.1$ to $n=2.7$ (this covers just about all commonly occurring dielectrics, except perhaps some precious gems which can have higher indices of refraction) the ratio $F_{\perp} / F_{\|}$is always above 3.0 , and often much higher, for the range of phase angles between $90^{\circ}$ and $130^{\circ}$. For all metals (not semiconductors) listed in [Physics Handbook] the ratio $F_{\perp} / F_{\|}$lies between 1.0 and 2.0 for the same range of phase angles. There is a big gap between 2.0 and 3.0 for the ratio $F_{\perp} / F_{\|}$between metals and dielectrics in the phase angle range between $90^{\circ}$ and $130^{\circ}$ !! What's more is that a metal or dielectric can be identified at a pixel point corresponding to a specular highlight by measuring $k_{\perp} / k_{\|}$. This approximates the Fresnel

\footnotetext{
${ }^{9}$ However the occurrence of a specular highlight may depend on surface orientation.
} 
ratio very well for metals, and dielectrics at specular angles of incidence away from the Brewster angle. For dielectrics using a specular angle of incidence equal to the Brewster angle, $k_{\perp} / k_{\|}$will be extremely large (i.e. $\gg 3$ ), underestimating an infinite Fresnel ratio.

This clear separation, between the values of the Fresnel ratio for metals and dielectrics, is important because it allows for reasonable inaccuracies in the above algorithm for estimating $F_{\perp} / F_{\|}$while minimizing the effect these errors have on the classification of materials. In particular, it is not important to know the exact light source location, nor is it necessary that $I_{d}$ be very close to zero (though it can not be too large). The constraint on the phase angle can also be slightly relaxed to be between $70^{\circ}$ and $150^{\circ}$ without too many misclassification problems. A future endeavor will be a more thorough theoretical analysis of the error characteristics of the above algorithm.

One of the advantages of this algorithm is that it can be implemented rather cheaply. Two images need to be acquired at different polarizations. ${ }^{10}$ Then the images are linearized and point by point differences can be computed. The image like parallism lends itself to implementation on fined grained or pipelined machines.

\section{CAMERA CALIBRATION AND EXPERIMENTAL TECH- NIQUE}

Important to the proper use of the above algorithm is the correction of the intensity images to accurately reflect scene radiance. The study of radiometric calibration for cameras has barely been touched, and yet it is essential to many model based feature extraction methods such as the one presented here. Some people such as [Shafer] are beginning to examine the many problems that are inherent to how a camera photosensor array converts scene radiance into an array of image irradiance values.

Unfortunately, current vision research is lacking a good study of the radiometric calibration of cameras. i.e., calibration of the relationship between photosensor response (i.e.. image irradiance) and the scene radiance, the camera optics and the polarization state of incident light. Fortunately, for the techniques presented herein, calibration of the absolute magnitude of incident radiance is not required because we only need to compute $k_{\perp} / k_{\|}$. However, to use equations 2 we must assume that the photosensor response is linearized. i.e. proportional to the scene radiance that enters the camera. Roughly, this means that if $a$ is radiance incident on a pixel giving gray level output $\alpha$, then if the scene is altered so that the incident radiance is $\delta a$, the output should become $\delta \alpha$. Unfortunately, this is rarely true for inexpensive video cameras. ${ }^{11}$

The theoretical typical response of a pixel photosensor is

$$
\text { image irradiance } \propto I^{1 / \gamma}
$$

where $I$ is the incident radiance and $\gamma$ is a constant which varies from device to device depending on whether the pixel is on a terminal monitor or a video camera etc. . Recommended values for $\gamma$ usually range from 2.0 to 3.0 [Foley and Van Dam 1982].

\footnotetext{
${ }^{10} \mathrm{~A}$ careful set up with a beam-splitter may be possible, but the set-up must insure that the splitting mirrors are not effecting the polarization state of the light.

11 This is what the gamma correction module of the camera is supposed to correct. But one must be careful, simply having the circuit there does not imply it is correctly linearizing the response. We also point out that many camera are actually designed to have a nonlinear response because this can be used to increase the camera's dynamic range, and having a nonlinear camera response and then doing the linearization/calibration yourself will give both a better dynamic range and a more accurate linearization.
} 
Rather than assume any specific value for $\gamma$, the camera was calibrated using a self-made variable neutral density filter from two Oriel model 27340 linear polarizers. The camera used was a Video Logic. Both linear polarizers were placed in tandem in front of the camera and the magnitude of the incident radiance is carefully controlled by varying the relative orientation of the two polarizers. Suppose at zero degrees relative orientation that the transmitted radiance of light through the two polarizers produces an image irradiance value $g_{\max }$. Most linear polarizers come with a specification of their extinction ratio. This is the ratio of the light transmitted through two of the linear polarizers in tandem when they are at $90^{\circ}$ relative orientation (minimum transmission) to the light transmitted when they are at $0^{\circ}$ relative orientation (maximum transmission). The extinction ratio for the polarizers we used are about 0.01 at most wavelengths within the visible spectrum. Therefore, if the gray values produced by pixel sensors are linear, and $g_{\max }$ is produced from the maximum transmission of the two polarizers in tandem, then the minimum transmission should produce gray value $g_{\min }=0.01 * g_{\max }$.

In [Siegel and Howell 1981] it is shown that the proportion of radiance incident on the camera when the relative orientations of the polarizers is $\theta_{2}$ to the incident radiance when the relative orientations of the polarizers is $\theta_{1}$ is given by

$$
\frac{\left(g_{\max }-g_{\min }\right) \cos ^{2} \theta_{2}+g_{\min }}{\left(g_{\max }-g_{\min }\right) \cos ^{2} \theta_{1}+g_{\min }}
$$

The camera was calibrated by keeping the inner polarizer fixed (i.e. the one closest to the camera) and rotating the outer polarizer at $15^{\circ}$ increments from zero degrees relative orientation to $90^{\circ}$ relative orientation. The relative orientation used for the denominator value was $\theta_{1}=0$. The camera was oriented toward the center of a diffusing plate for a uniform intensity field and a $25 \times 25$ pixel sample was taken at the center of the image. All image irradiance values were averaged over 32 images at the same pixel. The fstop was set at 2.8 and the focus was set at 3.5 feet. Of course all images of specular highlights were taken at the same settings.

Since most cameras are fairly sensitive to infrared light we also put a 300-800 NM pass filter on the camera. The peak value observed was $g_{\max }=236$ giving $g_{\min }=2.36$. Figure 5 shows a piecewise linear interpolation of the response curve determined by 8 sample points (including the measurement of absolute zero). The image irradiance value 236 is considered to be incident radiance level 1.0, and other incident radiance levels are relative to 1.0. A least squares approximation to the response curve yielded a $\gamma$ of about 2.1 .

The accuracy of the response curve in figure 5 is reduced by a number of possible problems for a video camera that have not been taken into account. For instance the photosensor response can be variable from one end of the pixel array to the other. Pixels away from the center of the pixel array are more sensitive to variations in the state of polarization of incident light. Effectively, only the center $25 \times 25$ pixel array was calibrated under the assumption that the same response holds everywhere else. The response of a given photosensor varies with respect to the spectral content of reflected light. Just because automatic gain control (AGC) is off does not mean that gain variations don't still occur for different lighting conditions. Also "charge bleeding" from one pixel photosensor to adjacent pixels can significantly effect the response curve. 


\section{EXPERIMENTAL RESULTS}

Figures 6 and 7 show experimental results for pictures of various metals and dielectrics. Figures 6 are of a teapot with a metal base and a plastic (dielectric) handle. Figures 7 show an assortment of objects; a roll of Aluminum foil, a strip of plastic leaning against the Aluminum roll, two coins, the larger a quarter and the smaller a copper penny, and a ceramic cup filled with water. The phase angle for the pictures in figures 6 using only the single light source was approximately $120^{\circ}$, and the phase angles for the different objects in the pictures in figures 7 , using the single light source, varied from $100^{\circ}$ to close to $90^{\circ}$. All pictures were taken at the same camera settings as for when the camera was calibrated. Again, all image irradiance values were averaged over 32 images at the same pixel. The white boxes emphasize which specular highlights were examined. Pictures taken with a single point light source are displayed as negatives so that the specular highlights could be visually discerned in the hardcopy of the displayed image.

Figure 6a shows the teapot illuminated with room lighting (i.e. an extended light source). Figures $6 \mathrm{~b}$ and $6 \mathrm{c}$ show the teapot illuminated with only a single light source for perpendicular and parallel orientations of an Oriel linear polarizer in front of the camera, respectively. Figure $6 \mathrm{~d}$ shows $4 \times 4$ pixel value arrays containing the peak values of the specular highlights within the white boxes in figures $6 \mathrm{a}-\mathrm{c}$. One of the highlights is on the metal base and the other highlight is on the plastic handle. The ratio $k_{\perp} / k_{\|}$was computed for pixels whose values using the perpendicular orientation were at least 50 . Note that the ratios for the highlight on the metal base lie between 1.0 and 2.0 , indicative of a metal. Note also that one of the ratios is actually less than 1.0. This is probably due to problems with camera gain (even though AGC was off) enhanced by "charge bleeding". This brings up a disadvantage with respect to using small specular highlights against a background of predominantly small pixel values. The charge on a small cluster of high pixel values will tend to "bleed off" into the lower pixel values or redistribute a bit amongst the high pixel values. Note the ratio $k_{\perp} / k_{\|}$for the highlight on the plastic handle of the teapot. This is very indicative of a dielectric. A number of the ratios were actually infinite, but any ratio over 10 is simply expressed $>10$.

The picture in Figure $7 \mathrm{a}$ was taken under extended room lighting using the perpendicular orientation of the polarizer in front of the camera. Figure $7 \mathrm{~b}$ shows the same scene under the same extended room lighting, but using a parallel orientation of the polarizer in front of the camera. Note that the metals and dielectrics in the scene can be qualitatively discerned by observing how some of the specular highlights vary. The highlights on the cup virtually disappear along with the specular highlight formed by the water on the side of the cup. The highlight on the plastic strip is very attenuated. The highlights on the coins and the Aluminum foil practically remain the same. Figures $7 \mathrm{c}$ and $7 \mathrm{~d}$ show the same scene for perpendicular and parallel orientations, respectively, of the polarizer in front of the camera, using a single light source. Figure $7 \mathrm{e}$ shows the values of $4 \times 4$ pixel arrays for the specular highlights. The computed ratios for $k_{\perp} / k_{\|}$ correspond very accurately to whether the highlight is on a dielectric or on a metal. Again, the ratio was computed for pixel values of at least 50 using the perpendicular orientation of the polarizer. In the absence of problems with gain and "charge bleeding", the ratios for the dielectrics should be even higher.

\section{CONCLUSION}

A potentially powerful new technique has been presented which discerns metal and dielectric material at pixels in an image. This technique is based upon the differing polarization properties of reflected light from metals and dielectrics. All that is required is the use of a linear polarizer (i.e. Polaroid filter) placed in front 
of the camera to resolve polarization components with respect to the specular plane of incidence, at each pixel. What makes this technique so robust is that it works independent of knowledge of the configuration of most imaging environments. Except for the constraint placed on the phase angle (i.e. between $90^{\circ}$ and $130^{\circ}$ ), no knowledge about imaging geometry or about surface orientation of an object is required. This technique equally applies to polished and rough surfaces. The technique works independent of light source color or intensity, and does not require an absolute magnitude calibration for a camera with respect to measuring scene radiance.

This technique was experimentally performed using a single point light source on a variety of common metal and dielectric surfaces, both rough and polished. All surfaces were correctly identified as being metal or dielectric at highlight regions where the specular component of reflection is dominant. The biggest disadvantage of this technique as it was performed in this paper is that it only works at specular highlight regions which usually cover a relatively small area on surface materials. By using extended lighting, the areas of dominant specular reflection on a material surface can be increased greatly, and this technique can be applied to a majority of points on the surface. There are other problems to overcome when using extended lighting such as the fact that in this case the specular plane of incidence is generally unknown and is not constant from point to point on the material surface. It turns out that images from at least three different orientations of a polarizer in front of the camera are required to provide an estimate of the Fresnel ratio when an extended source is used. This will be presented in future work.

\section{ACKNOWLEDGMENTS}

The author thanks his faculty advisor Terry Boult for reviewing this paper. The author also benefitted greatly from discussions with Steve Shafer about the nature of CCD cameras.

\section{References}

[Born and Wolf 1981]

[Buchanan 1987]

[Foley and Van Dam 182]

[Healey and Binford]

[Healey and Blanz 1988]

[Klinker et al. 1988]
Born, M., and Wolf, E., Principles of Optics, Sixth Edition, Pergamon Press, 1981.

Buchanan, C.S., Determining Surface Orientation From Specular Highlights, RCBV-TR-87-19, University of Toronto, Masters Thesis, August 1987.

Foley, J.D., Van Dam A., Fundamentals of Interactive Computer Graphics, Addison-Wesley, 1982, pp.594-597.

Healey, G., Binford, T.O. Predicting Material Classes, Proceedings of the DARPA Image Understanding Workshop, April 1988.

Healey, G., Blanz, W.E., Identifying Metal Surfaces In Color Images, SPIE 1988, April 1988, Orlando.

Klinker, G.J., Shafer, S.A., and Kanade T., The Measurement of Highlights in Color Images. International Journal of Computer Vision, Vol.2. No. 1, Spring, 1988. 
[Physics Handbook] American Institule of Physics Handbook, Third Edition, McGraw-Hill, 1972.

[Shafer] Steven Shafer. Personal communication.

[Siegel and Howell 1981] Siegel, R. and Howell, J.R., Thermal Radiation Heat Transfer, McGraw-Hill. 1981.

[Torrance and Sparrow 1967] Torrance, K. and Sparrow, E., Theory for Off-Specular Reflection from Roughened Surfaces. Journal of the Optical society of America, 57, pp.1105-1114, 1967.

[Wolf 1987] Wolff, L.B.. Surface Orientation From Polarization Images, SPIE Cambridge 1987, Optics, Volume 850, Illumination, and Image Sensing For Machine Vision II, pp.110-121. 
From Light Source

\section{To Viewer}

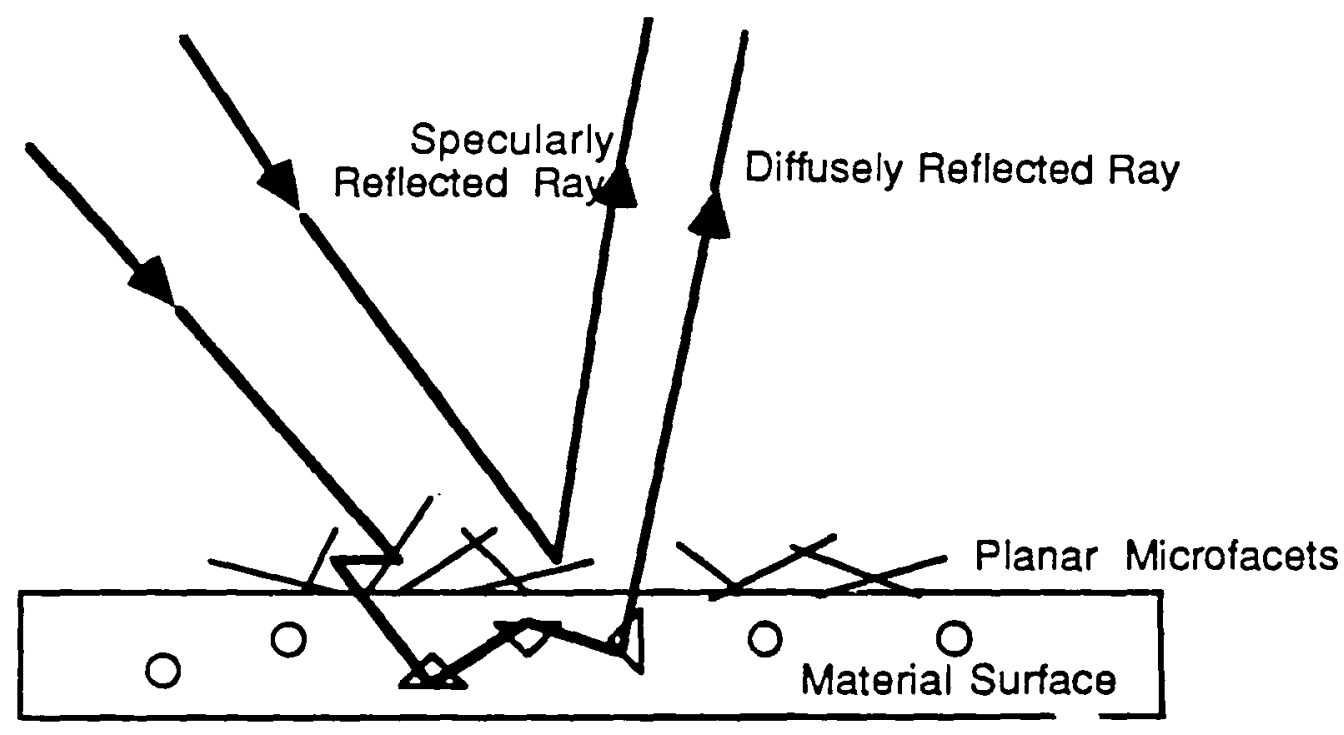

FIGURE 1 
WhFel:P

Suk:
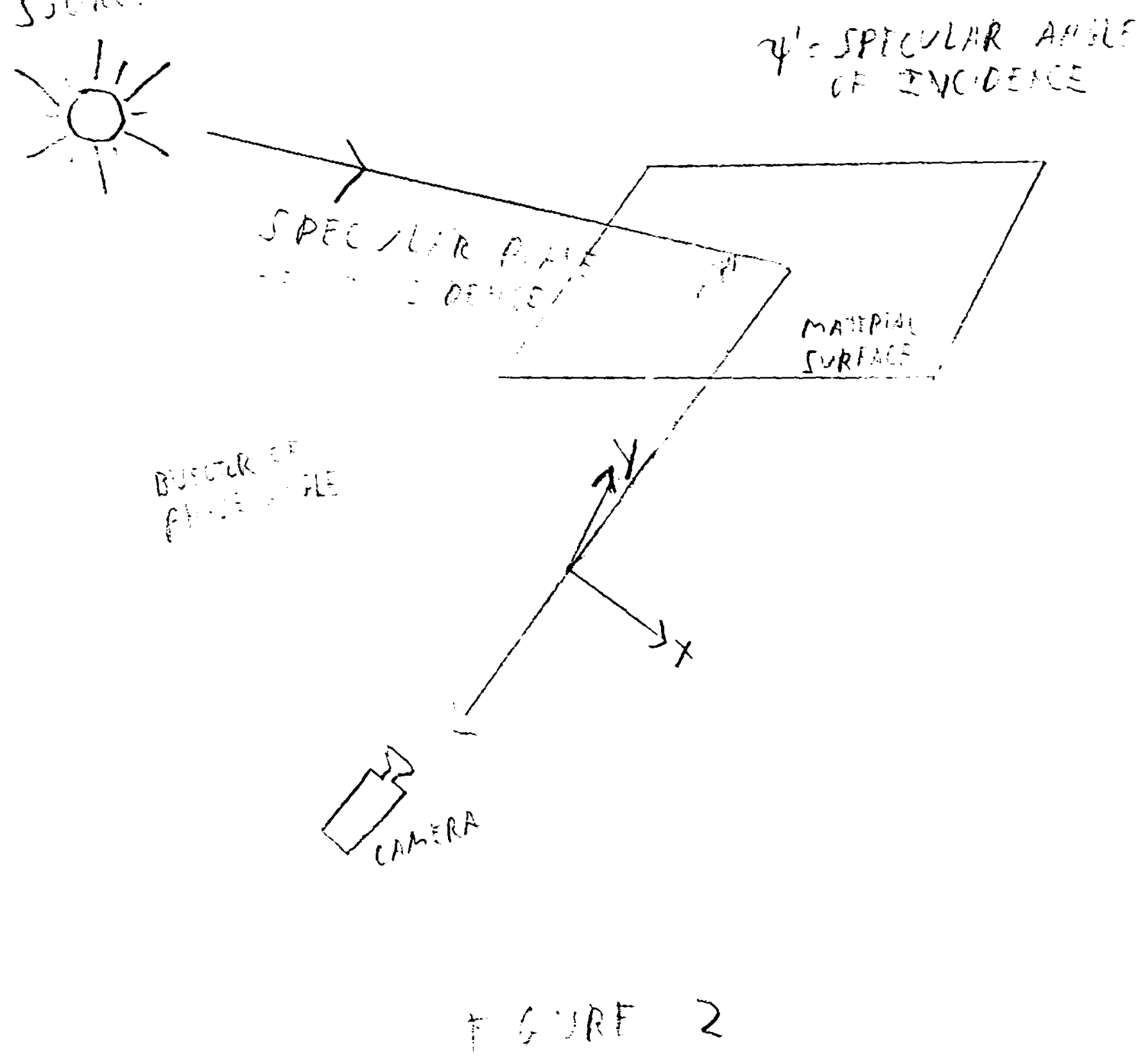


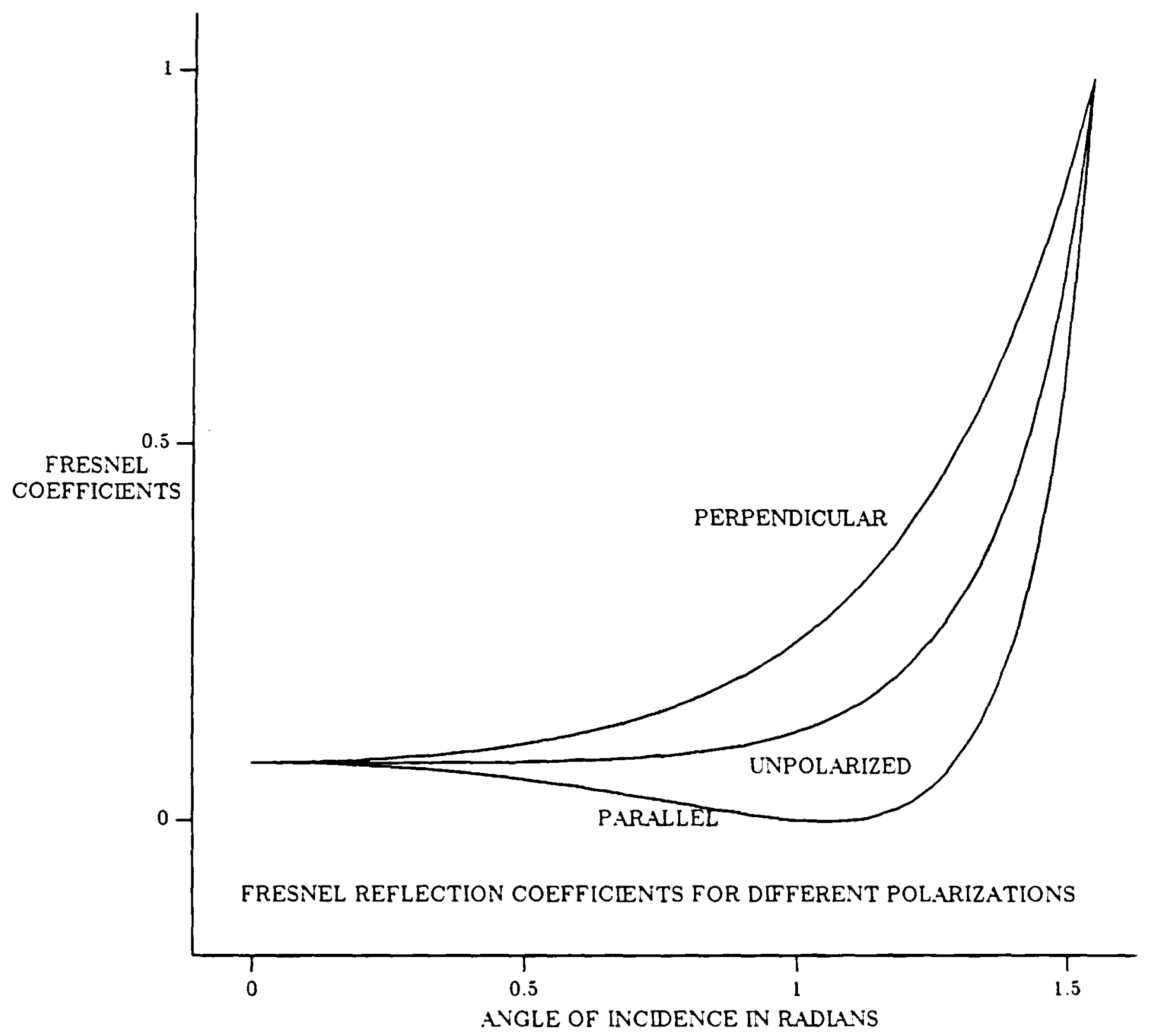

MLAGNESIUM O.UDE $\mathrm{N}=1.77 \mathrm{~K}=0.00$ COORRESPONDING TO REFLECTED LIGHT AT $365 \mathrm{NM}$

FIGURE 3 


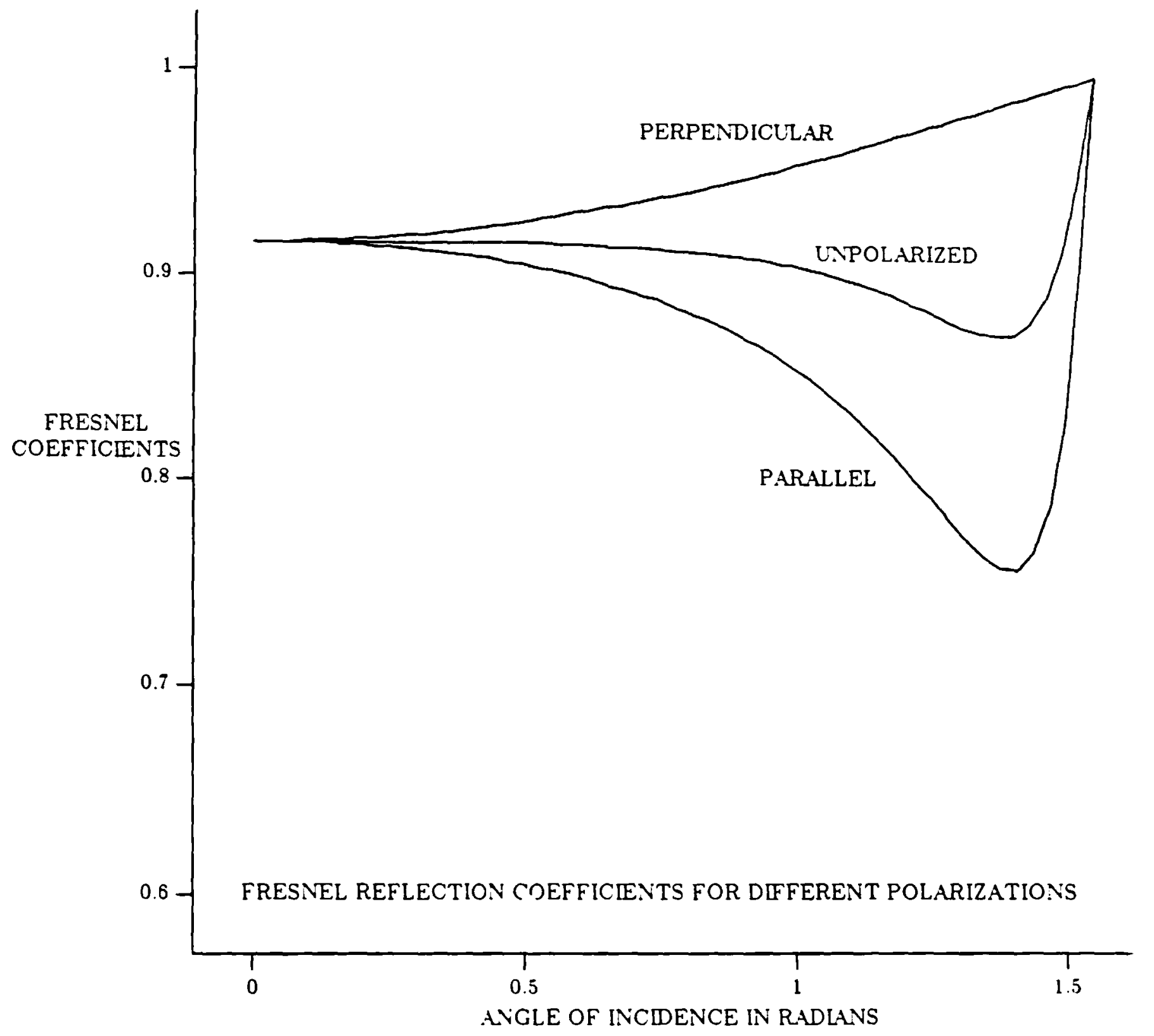

EVAPORATED ALUMINUM $\mathrm{N}=0.82 \mathrm{~K}=5.99$ CORRESPONDLNG TO REFLECTED LIGHT AT $546 \mathrm{NM}$

F. GURF 4 

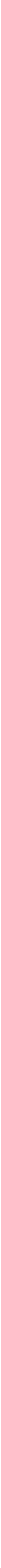
PIXEI VALUES AT SPECULAR HIGHIIGHTS FOR TEAPOT WITH HANDIE

HIGHIIGHT ON BASE OE TEAPOT

\section{PERPENDICULAR ORIENTATION}

$\begin{array}{lllll}70 & 129 & 110 & 71\end{array}$

$\begin{array}{llll}84 & 179 & 222 & 167\end{array}$

$\begin{array}{llll}145 & 237 & 233 & 225\end{array}$

$91 \quad 189208 \quad 121$

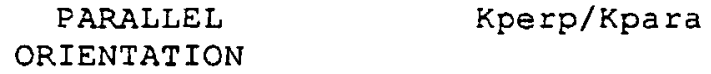

HIGHLIGHT ON HANDLE OE TEAPOT

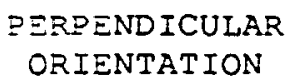

$\begin{array}{llll}6 & 8 & 60 & 129 \\ 13 & 126 & 191 & 78 \\ 82 & 93 & 67 & 55 \\ 25 & 27 & 24 & 6\end{array}$

\section{PARALLEL ORIENTATION}

$\begin{array}{llll}3 & 6 & 10 & 6 \\ 3 & 5 & 3 & 4 \\ 4 & 4 & 3 & 3 \\ 3 & 3 & 3 & 4\end{array}$

\section{Kperp/Kpara}

$-\quad-3.6>10$

$$
\text { - }>10>10>10
$$$$
>10>10>10>10
$$

$$
\text { Fibine } 6(d)
$$


SPECULAR HIGHLIGHT ON PLASTIC

. PERPENDICUIAR
ORIENTATION

$\begin{array}{lllll}75 & 195 & 234 & 180\end{array}$

$\begin{array}{llll}150 & 225 & 232 & 177\end{array}$

$\begin{array}{llll}134 & 215 & 210 & 137\end{array}$

$107 \quad 105 \quad 102 \quad 84$
PARALLEL ORIENTATION
Kperp/Kpara

$1.8 \quad 3.4 \quad 3.1 \quad 3.0$

$\begin{array}{llll}3.7 & 4.1 & 3.2 & 3.1\end{array}$

$4.4 \quad 4.9 \quad 3.9 \quad 4.2$

$4.5 \quad 4.4 \quad 4.2 \quad 3.6$

SPECULAR HIGHLIGHT ON ALUMINUM FOIL

\section{PERPENDICULAR ORIENTATION}

$\begin{array}{llll}115 & 144 & 112 & 60\end{array}$

$\begin{array}{llll}138 & 160 & 99 & 58\end{array}$

$\begin{array}{llll}101 & 98 & 56 & 97\end{array}$

$\begin{array}{llll}97 & 90 & 71 & 113\end{array}$

\section{PARALLEL ORIENTATION}

$\begin{array}{llll}102 & 140 & 118 & 61 \\ 132 & 163 & 114 & 56 \\ 97 & 108 & 59 & 87 \\ 91 & 97 & 68 & 103\end{array}$

Kperp/Kpara

$1.21 .0<1<1$

$1.1<1<1 \quad 1.0$ $1.1<1<1 \quad 1.2$ $1.1<1 \quad 1.11 .1$

SPECULAR HIGHLIGHT ON QUARTER

\section{PERPENDICUIAR ORIENTATION}

$\begin{array}{llll}50 & 53 & 52 & 57\end{array}$

$\begin{array}{llll}101 & 131 & 73 & 59\end{array}$

$\begin{array}{llll}73 & 158 & 192 & 70\end{array}$

$\begin{array}{llll}55 & 90 & 101 & 50\end{array}$
PERPENDICULAR ORIENTATION

$\begin{array}{llll}25 & 93 & 66 & 30 \\ 31 & 27 & 31 & 28 \\ 25 & 29 & 25 & 25 \\ 20 & 25 & 26 & 26\end{array}$

PERPENDICULAR ORIENTATION

$\begin{array}{llll}8 & 8 & 7 & 7 \\ 13 & 104 & 117 & 15 \\ 8 & 9 & 9 & 12 \\ 8 & 8 & 8 & 8\end{array}$

PERPENDICULAR
ORIENTAT ION
$\begin{array}{llll}13 & 9 & 9 & 8 \\ 46 & 29 & 15 & 9 \\ 9 & 47 & 90 & 39 \\ 7 & 7 & 7 & 8\end{array}$

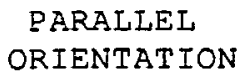

PARALLEL ORIENTATION

$\begin{array}{llll}38 & 40 & 38 & 41 \\ 88 & 103 & 58 & 39 \\ 67 & 138 & 186 & 82 \\ 43 & 69 & 99 & 49\end{array}$

1.21 .31 .31 .3

1.21 .41 .31 .4

$1.21 .31 .1<1$

1.21 .51 .01 .0

SPECUIAR HIGHLIGHT ON COPPER PENNY

\section{PARALLEL ORIENTATION}

$\begin{array}{llll}18 & 79 & 71 & 26 \\ 20 & 21 & 25 & 24 \\ 17 & 22 & 19 & 18 \\ 13 & 18 & 21 & 19\end{array}$

Kperp/Kpara

$\begin{array}{cccc}- & 1.3 & - & - \\ - & - & - & - \\ - & - & - & - \\ - & - & - & -\end{array}$

SPECULAR HIGHLIGHT ON CERAMIC CUP

\section{PARALLEI ORIENTATION}

$\begin{array}{llll}7 & 7 & 6 & 7 \\ 10 & 16 & 17 & 9 \\ 7 & 8 & 8 & 11 \\ 8 & 8 & 7 & 7\end{array}$

SPECULAR HIGHLIGHT ON WATER

$$
\begin{aligned}
& \text { BARAILEL } \\
& \text { ORIENTATION } \\
& \begin{array}{llll}
12 & 9 & 8 & 8 \\
25 & 17 & 11 & 9
\end{array} \\
& \begin{array}{llll}
10 & 22 & 30 & 22 \\
7 & 6 & 7 & 8
\end{array} \\
& \text { figure } 7(e)
\end{aligned}
$$

